\title{
Anti-ICOS Monoclonal Antibody KY1044
}

National Cancer Institute

\section{Source}

National Cancer Institute. Anti-ICOS Monoclonal Antibody KY1044. NCI Thesaurus. Code C162521.

A human immunoglobulin G1 (IgG1) kappa monoclonal antibody that recognizes inducible T-cell co-stimulator (ICOS; CD278), with potential immunomodulating and antineoplastic activities. Upon administration, anti-ICOS monoclonal antibody selectively binds to dimeric ICOS expressed on certain T-cells. This prevents the interaction between ICOS-positive T-cells and plasmacytoid dendritic cells (pDCs), which express the ICOS ligand (ICOSL). Blocking ICOS activation prevents the pDC-induced proliferation and accumulation of regulatory ICOS-positive T-cells (ICOS+ Tregs) and inhibits interleukin-10 (IL-10) secretion by CD4+ infiltrating T-cells. This may abrogate Treg-mediated immune suppression and may enhance cytotoxic T-lymphocyte (CTL)-mediated immune responses against tumor cells. Additionally, KY1044 may eliminate ICOS-positive T-cells via antibody-dependent cellular cytotoxicity (ADCC). ICOS, a T-cell specific, CD28-superfamily costimulatory molecule and immune checkpoint protein, plays a key role in the proliferation and activation of T-cells. It is normally expressed on both activated CD4+ T-cells, which is a subset of memory T-cells (T m), and follicular helper T-cells (Tfh). ICOS is highly expressed on Tregs infiltrating various tumors and its expression is associated with a poor prognosis; ICOS-positive Tregs play a key role in immune suppression and tumor immune evasion. 